\title{
Ocular inflammatory changes in established multiple sclerosis
}

\author{
E M GRAHAM, D A FRANCIS, M D SANDERS, P RUDGE \\ From the National Hospital for Nervous Diseases, Queen Square, London
}

SUMMARY Fifty consecutive patients with clinically definite multiple sclerosis were studied to assess the prevalence of concomitant uveitis. Asymptomatic ocular inflammatory changes were found in nine patients $(18 \%)$ and appeared to show a positive correlation with severe and progressive disease. Conversely uveitis was uncommon in the presence of established optic atrophy which suggests a negative influence on its pathogenesis. In the absence of optic atrophy inflammatory changes in the eye may be a valuable index of disease activity.

Ocular inflammatory changes have been a recognised occurrence in multiple sclerosis (MS) for many years, ${ }^{1}$ although their pathogenetic significance remain uncertain. Recent studies have found associations between retinal vascular abnormalities and both the later development of MS in patients presenting with isolated optic neuritis $(\mathrm{ON})^{2}$ and neurological disease activity in patients with established MS. $^{3}$

In this study we have sought the prevalence of ocular inflammatory changes (uveitis) in patients with clinically definite MS and correlated their presence with clinical course and disease severity. Our findings may shed further light on their occurrence and pathogenesis.

\section{Patients and methods}

We studied consecutive patients with clinically definite MS but without preceding ocular disease (see below), ${ }^{4}$ who were admitted to one neurological firm at the National Hospital, Queen Square, London. Patients with a past history of ocular disease, other than optic neuritis, or systemic illness associated with ocular abnormalities were excluded. A total of 50 patients was collected, 27 were female and 23 male. Their mean age at presentation of first neurological symptoms was $28 \cdot 3$ years (range $16-51$ years).

Patients were categorised according to the severity (mild, moderate or severe) and clinical course (remittent or currently progressive) of their disease. Patients with mild disease had a score of $\leqslant 3$ on the Kurtzke disability scale, ${ }^{5}$ moderate disease 3.5-6 and severe disease $>6$ at the time of assessment.

Correspondence to: Dr D A Francis, National Hospital for Nervous Diseases, Maida Vale, London W9 1TL, United Kingdom.

Received 10 March 1989 and in revised form 9 June 1989. Accepted 17 July 1989
Patients with currently progressive MS had shown either a progressive evolution of disease from onset or had entered a progressive phase following initial remissions.

All fifty patients had a full ophthalmological examination. This included assessment of corrected visual acuity (Snellen), colour vision (Ishihara plates), visual fields (Bjerrum screen) slit-lamp examination and direct and indirect ophthalmo scopy. Fluorescein angiography was performed only when clinically indicated. Ocular inflammation (uveitis) was defined by the presence of inflammatory cells in the vitreous with either sheathing of peripheral retinal vessels and/or foca cuffing of retinal veins (periphlebitis).

\section{Results}

\section{Neurological features}

The neurological features are summarised in table 1 . The mean duration of MS for the group as a whole was 12.3 years (range $0.5-38$ years); fifty per cent of patients had had neurological symptoms for 10 years or more. Thirty four patients $(68 \%)$ had remittent disease and in 16 patients $(32 \%)$ the disease was currently progressive. The age at onset and disease

Table 1 Clinical features of 50 consecutive patients with $M S$

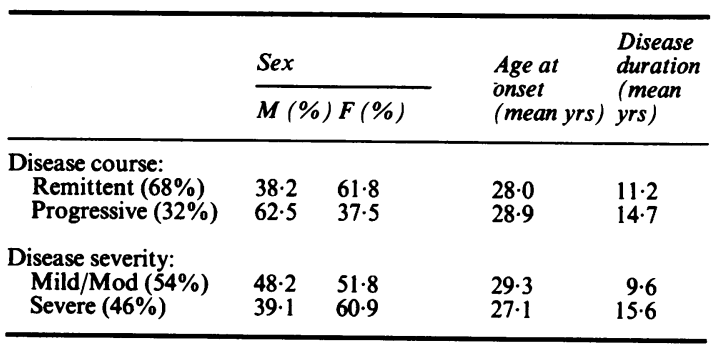


Table 2 Neurological status of patients with ocular inflammation (uveitis)

\begin{tabular}{lccc}
\hline \multicolumn{2}{l}{ Neurological status } & \multicolumn{2}{c}{ Uveitis \% } \\
\hline Overall & $(50)$ & $18 \cdot 0$ & $(9)$ \\
Remittent & $(34)$ & 11.8 & $(4)$ \\
Progressive & $(16)$ & $31 \cdot 3$ & $(5)$ \\
Mild & $(12)$ & $8 \cdot 3$ & $(1)$ \\
Moderate & $(15)$ & 20.0 & $(3)$ \\
Severe & $(23)$ & $21 \cdot 7$ & $(5)$ \\
Disease duration: & & \\
$>10$ years (25) & 28.0 & $(7)$ \\
$<10$ years (25) & 8.0 & $(2)$ \\
\hline
\end{tabular}

$(\quad)=$ Number of patients.

duration were similar in both groups, although females tended to follow a remittent course and males a more progressive evolution. Twenty seven patients (54\%) had disease of mild to moderate severity; 23 patients $(46 \%)$ severe MS. Females predominated in the severe category but overall had had their disease for a longer period.

\section{Ophthalmoscopic features}

Nine patients (18\%; five males, four females) had evidence of ocular inflammation (venous sheathing in three; focal venous cuffing in four) at the time of ophthalmoscopic examination. Their neurological status is summarised in table 2; ocular inflammatory changes were present in $31.2 \%$ of patients with progressive disease (five patients), $21.7 \%$ of patients with severe MS (five patients) and $28 \%$ of patients with disease of more than 10 years duration (seven patients).

In eight patients the inflammatory changes were present in both eyes. In one patient it was unilateral; the other eye, showing marked optic atrophy secondary to previous trauma, had no signs of uveitis. This patient underwent fluorescein angiography (fig 1) and further electrodiagnostic studies. The visual evoked responses (VER) from both eyes were significantly delayed (right eye, $145 \mathrm{msec}$; left eye, $175 \mathrm{msec}$; normal range 95-117 msec). The electro-oculogram (EOG) and the electroretinogram (ERG) also showed bilateral abnormalities although conversely the right eye was marginally worse than the left. The EOG light-rise on the right was $200 \%$ and on the left $220 \%$ (NR 250 $300 \%$ ); the averaged cone ERG was $5.8 \mu \mathrm{v}$ on the right and 7.0 $\mu \mathrm{v}$ on the left (NR 8-9 $\mu \mathrm{v}$ ).

Visual function appeared better preserved in patients with uveitis thus visual acuity was $6 / 9$ or better in all 17 eyes with active inflammation compared to $80 \%$ of unaffected eyes; colour vision was impaired in only 1/17 "affected" eyes compared to $(75 \%)$ of the remainder and optic atrophy was present in only six of 17 eyes showing inflammatory changes
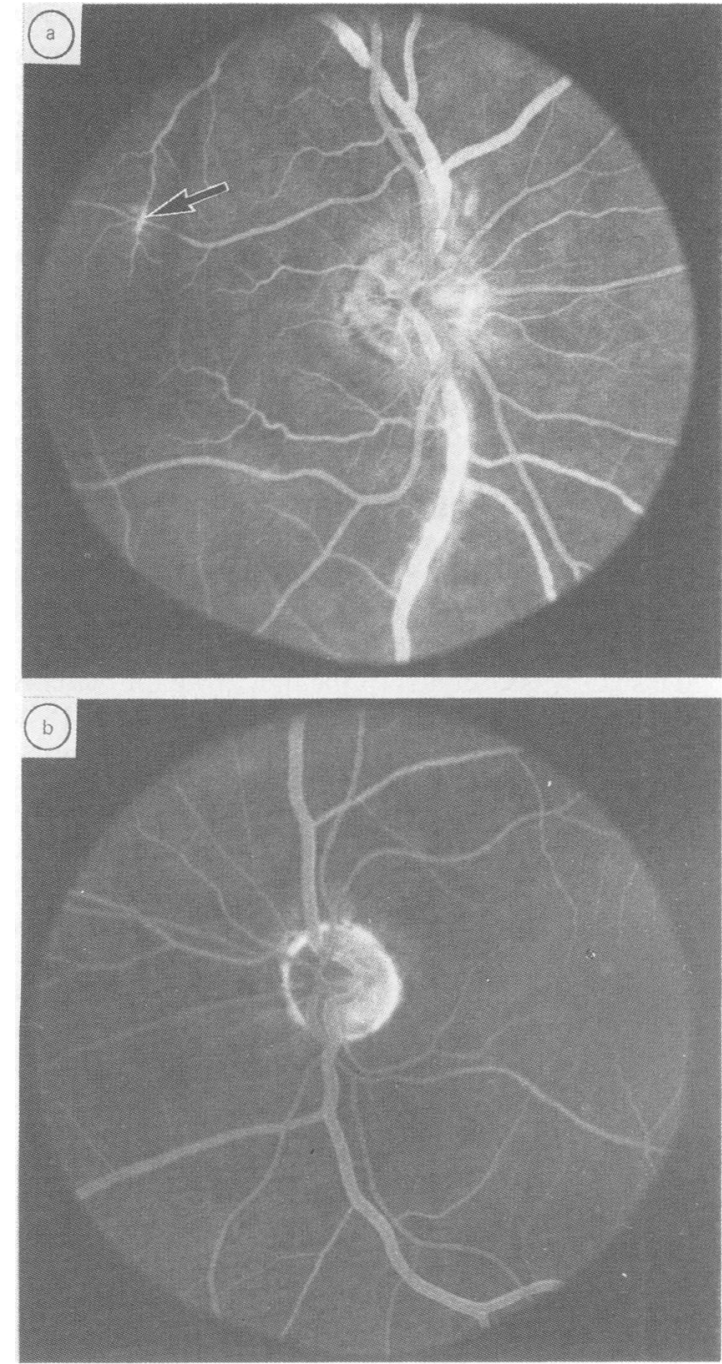

Fig 1 (a top). Late fluorescein angiogram of right eye showing dilated retinal veins with perivascular leakage (arrow). ( $b$ bottom). Late fluorescein angiogram of left eye which does not show any evidence of vascular leakage.

(35\%) compared to $46 / 81(55 \%)$ of unaffected eyes (chi-square $=2 \cdot 6$ ).

\section{Discussion}

The combination of retinal vascular abnormalities and neurological disease is seen in three distinct groups of patients. Firstly, those that Rucker' identified with MS who have established neurological disease with no symptoms referable to ocular inflammation, but in whom routine ophthalmological examination reveals 
cells in the vitreous, peripheral venous sheathing and/ or focal cuffing of the retinal veins. Secondly patients present complaining of "floaters" across their vision which are caused by inflammatory cells in the vitreous; these patients also have sheathing of their peripheral retinal vessels and diffuse vascular leakage demonstrated on fluorescein angiography. A recent study of 65 patients with isolated retinal vasculitis has revealed that $10 \%$ later developed MS. ${ }^{6}$ The third group of patients have symptomatic ocular and neurological disease at presentation. The eye disease is often termed "Eales disease" and is characterised by severe periphlebitis, retinal vein occlusion leading to neovascularisation and vitreous haemorrhage. The neurological component is usually monophasic and most often consists of a spastic paraparesis. ${ }^{89}$ Multiple sclerosis is a rare cause of this combination whereas tuberculosis, sarcoidosis or the systemic vasculitides are frequently associated.

This study is confined to the first group of patients defined above in which we have found an $18 \%$ prevalence of ocular vascular abnormalities, consistent with uveitis, in patients with established MS. This figure is comparable to other hospital based series ${ }^{310}$ but contrasts with recorded prevalences of only $1 \%$ $5 \%$ noted among patients with quiescent MS attending rehabilitation centres. ${ }^{3}$ However, all nine patients in our study with ocular changes had active neurological disease; this concords with the studies of Bamford et al ${ }^{11}$ and Engell ${ }^{3}$ in which $20-40 \%$ of patients with a severe or progressive course had coexistent uveitis. Together these findings support the concept that ocular inflammatory changes may represent the visible correlate of concomitant active CNS demyelination. There are histological and antigenic similarities between retinal and cerebral vascular endothelium which may be important in the early stages of demyelination. These similarities are discussed fully elsewhere. ${ }^{212}$

One question which remains unanswered is why not all patients with active MS show similar retinal changes. It is possible that asymptomatic retinal vascular inflammation is a transient phenomenon occurring only at the onset of an acute neurological relapse and therefore missed by the unsuspecting physician. In addition, in many cases the inflammatory changes are in the most peripheral vessels and only seen with the indirect ophthalmoscope. We would like to propose a third explanation. One patient in our series showed signs of florid ocular inflammation in one eye only, the other eye, with pronounced optic atrophy following previous trauma, was unaffected. We also noted that uveitis was clinically more pronounced in those eyes unaffected by optic atrophy. It is generally accepted that severe optic atrophy appears to "protect" against ocular inflammation.
For example patients with Behçet's disease who experience recurrent uveitis with retinal vascular occlusion will eventually develop optic atrophy; at this stage the ocular disease process is arrested and uveitis never recurs (EMG unpublished data).

The mechanism of this phenomenon is unclear but there are at least two possible explanations. One is to assume that severe optic atrophy reduces retinal vascularity and lessens the opportunity for immunological reactions to take place at the vascular endothelium. The second is that, if a component of CNS tissue is the prime immunological target, the inflammatory reaction would be expected to subside with its progressive loss. The electrodiagnostic and fluorescein studies in our patient with traumatic left optic atrophy confirmed that optic nerve damage was the predominant pathological feature in the left eye whereas retinal damage was more pronounced in the right eye. Retinal photoreceptor damage is known to occur as a result of retinal vasculitis ${ }^{13}$ and this is borne out by the asymmetrical ERG responses we obtained; the ERG from the "unaffected" left eye indicated almost intact photoreceptor function. These findings would therefore seem to support the former explanation that $\vec{N}$ the development of ocular inflammation requires añ $\vec{\omega}$ adequate blood supply. The retinal perivasculaf ${ }_{0}$ abnormalities seen in patients with MS may thue 음 represent a visible manifestation of active inflammae tion occurring within the CNS but which is potentiall masked by optic atrophy occurring secondary to $\overbrace{0}^{\circ}$ clinical or subclinical optic neuritis. Other inveso $\frac{\Phi}{3}$ tigators have noted a negative correlation betweef "retinal sheathing" and optic atrophy in $\mathrm{MS}^{1}$ but oup observations require more detailed confirmation before firm conclusions can be made.

We are grateful to Drs C J Earl and K Zilkha for allowing us to study their patients and to Professor $\mathrm{H}$ Ikeda for performing the electrophysiological study.

\section{References}

1 Rucker CW. Sheathing of the retinal veins in multiple sclerosis. Mayo Clinic Proc 1944;19:176-8.

2 Lightman S, McDonald WI, Bird AC, et al. Retinal venous sheathing in optic neuritis. Brain 1987;110: 405-14.

3 Engell T. Neurological disease activity in multiple sclerosis patients with periphlebitis retinae. Acta Neurol Scand 1986;73:168-72.

4 Poser CM, Paty DW, Scheinberg L, et al. New diagnostic criteria for multiple sclerosis: guidelines for research $\bigcirc$ protocols. Ann Neurol 1983;13:227-31.

5 Kurtzke JF. On the evaluation of disability in multiple $\frac{7}{2}$ sclerosis. Neurology (Minneap) 1961;11:686-94.

6 Sanders MD. Retinal arteritis, retinal vasculitis and $\mathbb{N}$ autoimmune retinal vasculitis. Eye 1987;1:441-65. 
7 Eales H. Primary retinal haemorrhage in a young man. Opthal Rev 1882;1:41.

8 Silverskiold BP. Retinal periphlebitis associated with paraplegia. Arch Neurol Psychiatry 1947;57:351-4.

9 Singhal BS, Dastur DK. Eales disease with neurological involvement. Part 1: Clinical features in 9 patients. $J$ Neurol Sci 1976;27:313-21.

10 Moller PM, Hammerberg PE. Retinal periphlebitis in multiple sclerosis. Acta Neurol Scand 1963;39(suppl
4):263-9.

11 Bamford CR, Ganley JP, Sibley WA, Laguna JF. Uveitis, perivascular sheathing and multiple sclerosis. Neurol (NY) 1978;28:119-24.

12 Rahi AHS, Garner A. Immunopathology of the eye. Oxford: Blackwell, 1976:221-34.

13 Stanford MR, Brown EC, Kasp E, et al. Experimental posterior uveitis. I: A clinical, angiographic and pathological study. Brit J Ophthalmol 1987;71:585-92. 